\title{
Design and analysis of electrical automation control system based on path optimization hybrid algorithm
}

\author{
Su Hongyu \\ Harbin Cambridge University, Harbin, 150069, China, 20130602@stu.nmu.edu.cn, Corresponding author
}

\begin{abstract}
With the rapid development of science and technology, the application of various electrical equipment is becoming more and more common, and people's application of electrical automation is becoming more and more extensive. Using intelligent automation equipment can not only improve production efficiency, but also help us solve many life problems. In order to improve the control accuracy of electrical automation control system, an electrical automation control system based on path optimization hybrid algorithm is proposed. In this system, the expert intelligent control algorithm is selected to obtain the operating parameters of electrical equipment in real time, and the idea of combining genetic algorithm (GA) with simulated annealing algorithm is adopted to prevent premature phenomenon, ensure the diversity of population and prevent local optimization. Finally, the simulation results show that the hybrid algorithm is feasible and scientific. Finally, it is concluded that the optimization efficiency and quality of the new algorithm are better than those of basic ACA and GA.
\end{abstract}

\section{CCS CONCEPTS}

- Information systems $\rightarrow$ Information systems applications.

\section{KEYWORDS}

Genetic algorithm, Ant colony algorithm, Path optimization hybrid algorithm, Electrical automation control

\section{ACM Reference Format:}

Su Hongyu. 2021. Design and analysis of electrical automation control system based on path optimization hybrid algorithm. In 2021 4th International Conference on Information Systems and Computer Aided Education (ICISCAE'21), September 24-26, 2021, Dalian, China. ACM, New York, NY, USA, 5 pages. https://doi.org/10.1145/3482632.3482744

\section{INTRODUCTION}

The electrical automation control system is a comprehensive control management system based on computer technology and electronic communication technology. Intelligent technology is a cutting-edge technology developed in the field of computer, which can replace manual work by computer programs [1]. Although China's electrical automation control technology has made great achievements, compared with western developed countries, China's electrical

Permission to make digital or hard copies of all or part of this work for personal or classroom use is granted without fee provided that copies are not made or distributed for profit or commercial advantage and that copies bear this notice and the full citation on the first page. Copyrights for components of this work owned by others than ACM must be honored. Abstracting with credit is permitted. To copy otherwise, or republish, to post on servers or to redistribute to lists, requires prior specific permission and/or a fee. Request permissions from permissions@acm.org.

ICISCAE'21, September 24-26, 2021, Dalian, China

(C) 2021 Association for Computing Machinery.

ACM ISBN 978-1-4503-9025-5/21/09 . \$ \$15.00

https://doi.org/10.1145/3482632.3482744 automation control technology is still in a weak position. With the gradual maturity of China's market economy, the frequency of international communication increases, and the competition of electrical automation control technology becomes more and more intense [2]. Because the system wiring of centralized monitoring mode is complex, there are some problems such as difficult line checking and tedious operation and maintenance. Therefore, we must pay more attention to the system wiring.

Research shows that genetic algorithm (GA) is a common algorithm to solve the shortest path, and as an effective global parallel search tool, it is simple, universal, robust and suitable for parallel distributed processing [3]. However, it has obvious defects in some aspects: it is prone to precocity and other phenomena; Due to the adoption of random search algorithm, the convergence speed decreases and the similarity increases. Therefore, this paper designs an electrical automation control system based on path optimization hybrid algorithm to improve the electrical automation control effect.

\section{DESIGN OF ELECTRICAL AUTOMATION CONTROL SYSTEM}

\subsection{External environmental impact analysis}

The external environment of electrical automation equipment is generally complex, which has a certain influence on the performance and life of the equipment. It is necessary to strengthen the rational control of external environment and realize the optimal utilization of equipment. The influence of external natural environment can't be ignored, such as mold, high humidity, serious pollution and other negative environmental influences, which will have adverse effects on equipment, so it is necessary to strengthen the prevention, control and management of equipment erosion.

It is necessary to strengthen the prevention, control and management of equipment erosion, avoid the increase of electrical conductivity of insulating materials caused by moisture and special substances, and improve equipment performance, so as to fully realize the goal of long-term stable and continuous operation, improve the rationality of power plant automation control system, and promote the steady growth of economic benefits of enterprises. Therefore, it is crucial to control the external environmental factors reasonably from the perspective of electrical automation control equipment.

\subsection{Line design system}

The related circuits of the electrical design system are complex and must be operated by professionals. The quality of circuit design will directly affect the function of the whole power supply system, and circuit design is also one of the key links in electrical design. Designers can use the line design system to produce drawings with perfect information. This step requires designers to input relevant 


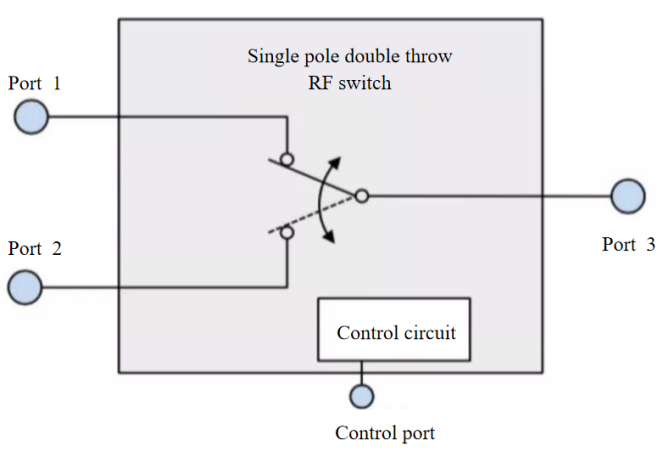

Figure 1: Principle and construction of filter

information into the line design system, such as engineering materials consumed in design, actual road conditions of the project, etc. This series of work is an essential link in line design. After the designer makes the drawings, the system can generate data independently, and these data will change with the factors of line design. The line design system can provide the designer with the actual situation of the line, which is convenient for the staff to adjust and modify the plan in time.

Under the present situation, the use and application of its automation equipment should be operated in a relatively complex environment. This severe environmental factor will cause great damage and obstacles to the application of equipment. Under the current technical conditions, there are two alternative ways to purify the power supply. These two devices are called filter and isolation transformer device respectively [4]. Figure 1 is used to show the working principle of the filter and the related pattern composition.

Using relays to carry out related output work, this output mode can reduce the interference caused by environment and various factors and reduce the load phenomenon, making it safer and more efficient. In addition, when related PLC output is carried out, it will sometimes cause surge current and damage PLC equipment. To avoid this situation, it is suggested to connect diodes on the circuit board, which can absorb surge current and protect the normal operation and safety of PLC chips.

\subsection{Electrical fault intelligent diagnosis module}

Intelligent electrical fault diagnosis can comprehensively check the running status of electrical equipment, monitor the real-time working parameters of equipment, collect relevant data and information, and judge the type and location of faults. And then fed back to the host computer of the system, which is convenient for the maintenance of managers. The flow chart of the designed electrical fault diagnosis module is shown in Figure 2

The main principle of this intelligent diagnosis method is to save the data parameters of each device in the system database in advance when the control system runs normally [5]. In the later operation, on the one hand, the intelligent control system dynamically obtains the current operating parameters of electrical equipment and systems in real time.

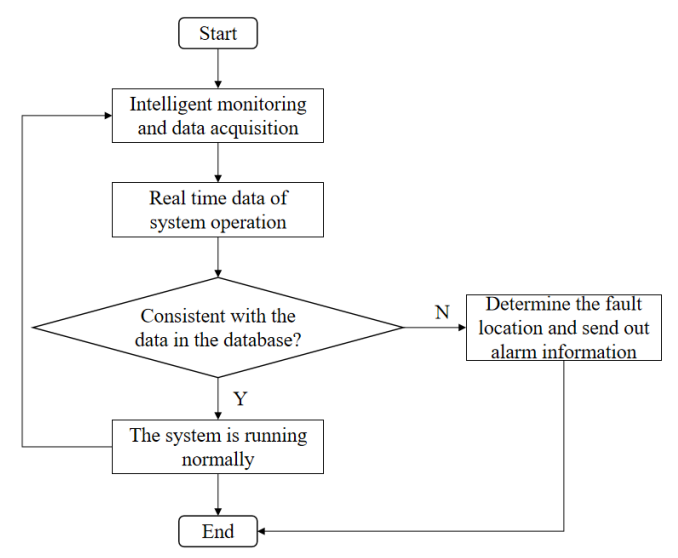

Figure 2: Operation flow chart of diagnostic module

Compare these data with the data information previously saved in the database. If the data is within the error range, all parts work normally; Otherwise, find the inconsistent data, judge the data source, and then determine the fault location, which provides an important reference for the subsequent operators.

\subsection{Analysis of equipment heat dissipation protection treatment}

As the guarantee of electrical automation control, standby heat dissipation protection treatment has a prominent influence on the performance parameters and service life of components. Therefore, strengthening the reasonable protection and management of equipment has a positive impact on the stability and safety of the system, and at the same time can extend the application period of the equipment. Achieve the goal of maximizing the economic benefits of the control system, reduce the operating costs of enterprises, and drive the steady increase of economic benefits.

Equipment heat dissipation protection has a prominent influence on the later system operation. In order to avoid equipment damage and system paralysis, it is necessary to strengthen the optimized control treatment of equipment protection, avoid the hidden trouble caused by heat dissipation failure, and strengthen the effective discharge treatment of heat generated under normal working conditions, especially the heat dissipation control management of high-power equipment. In addition, it should be noted that the use of sensitive conductor materials should be avoided as far as possible around high-power equipment, which has a positive impact on eliminating hidden dangers.

\section{CONTROL STRATEGY BASED ON PATH OPTIMIZATION HYBRID ALGORITHM}

\subsection{Ant colony system}

The existing research results have shown that the ant colony optimization algorithm has a strong ability to find the best solution, can perform multi-point calculation, is easy to combine with other algorithms, and has simple running conditions and is easy to set [6]. However, it also has some shortcomings, such as lack of initial 
pheromones, long search time, and easy to fall into local optimal solution when the scale is large [7]. In order to overcome these shortcomings of the basic ant colony algorithm (ACA), people studied and improved it.

Ant colony system(ACS) is an improved version of AS (ant system) algorithm. The main difference between ACS and ant system lies in:

(1)When choosing the next city first, ACS algorithm makes more use of the current better solution.

(2)Only the pheromone is added on the edge where the global optimal solution belongs.

(3)Every time ants move from cityito city $j$, pheromones on edge $i j$ will reduce path construction appropriately.

In ACS, ant $k$ in cityichooses city $j$ as the next city to be visited according to the pseudo-random proportional rule, which is given by the following formula.

$$
\begin{aligned}
& j=\left\{\begin{array}{l}
\underset{u \in \text { allowed }_{k}}{\arg \max }\left[t_{i u}(t)\right]\left[h_{i l}\right]^{b} \quad q \leq q_{0} \\
S \quad q \leq q_{0}
\end{array}\right. \\
& p_{i j}^{k}(t)=\left\{\begin{array}{l}
\frac{\left[\tau_{i j}(t)\right]^{\alpha}\left[\eta_{i j}(t)\right]^{\beta}}{\sum_{s \in \text { allowed }_{k}}\left[\tau_{i s}(t)\right]^{\alpha}\left[\eta_{i s}(t)\right]^{\beta}} \quad j \in \text { allowed }_{k} \\
0 \quad \text { other }
\end{array}\right.
\end{aligned}
$$

In which $q$ is a random variable uniformly distributed in the interval $[0,1]$, and $q_{0}\left(0 \leq q_{0} \leq 1\right)$ is a parameter (where $\alpha=1$ ).

Global partial pheromone update, in ACS, only one ant (the current optimal ant) is allowed to release pheromone after each iteration. In this way, the pheromone update rule of ACS is given by the following formula:

$$
\begin{gathered}
\tau_{i j}=(1-\rho) \tau_{i j}+\rho \Delta \tau_{i j}^{b s}, \quad \forall(i, j) \in T^{b s} \\
\Delta \tau_{i j}^{b s}=\frac{1}{C^{b s}}
\end{gathered}
$$

In addition to global pheromone update, ACS also adopts a local pheromone update rule. In the process of path construction, ants will immediately call this rule to update the pheromones on an edge every time they pass through $(i, j)$ :

$$
\tau_{i j}=(1-\rho) \tau_{i j}+\xi \Delta \tau_{0}
$$

In which $\xi$ and $\tau_{0}$ are two parameters, $\xi$ satisfies $0<\xi<1$, and $\tau_{0}$ is the initial value of pheromone quantity. In the experiment, it is found that when $\xi$ is 0.1 and $\tau_{0}$ is $1 / C^{n n}$, the algorithm has better results. $n$ represents the number of cities, and $C^{n n}$ represents the length of the path obtained by the nearest neighbor method. The function of local updating is to reduce the pheromone amount $\tau_{i j}$ of an edge every time an ant passes through the edge $(i, j)$, thus reducing the relative probability of other ants selecting the edge [8].

\subsection{PID and fuzzy switching control}

PID control is one of the widely used methods in industrial control, because it has the advantages of strong stability and high reliability, but the anti-interference ability of this control method is insufficient, especially in the continuous casting production system, the production phenomenon has the characteristics of strong interference and time variability, which is not conducive to the accurate

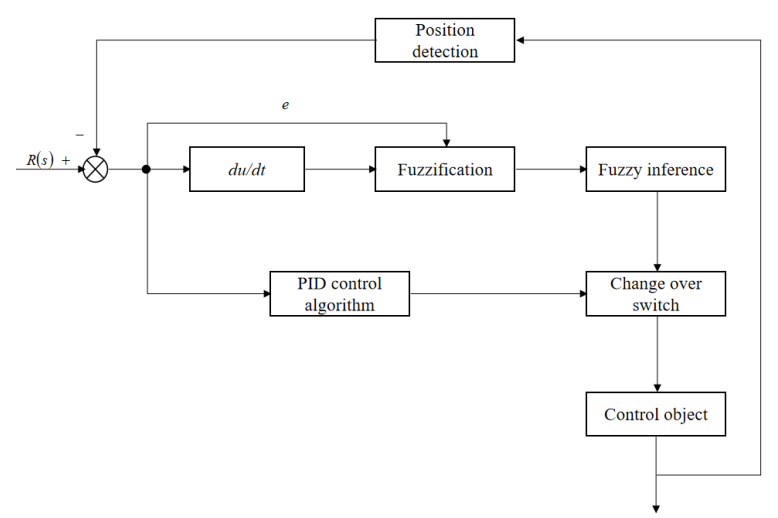

Figure 3: Structure model of PID and fuzzy switching controller

establishment of mathematical model, so the effect of adopting automatic control algorithm is not satisfactory [9].

In PID and fuzzy switching control system, PID controller can obtain better steady-state performance, while fuzzy controller can obtain better dynamic performance. Because the deviation change rate and deviation value of the system are important parameters that determine the output of PID and fuzzy switching controller, it is necessary to analyze these parameters. When the system deviation is small, the system chooses PID controller, and when the system deviation is large, the system can choose fuzzy controller, thus completing the accurate control of the system in the process of controller switching. The structure model of PID and fuzzy switching controller is shown in Figure 3

Select PID controller as the controller to control the system, and its transfer function is

$$
G(s)=K_{p}\left(1+\frac{1}{T_{i} s}\right)+T_{d} s
$$

In the above formula, $T_{d}$ is a differential time constant, $T_{i}$ is an integral time constant, and $K_{p}$ is a proportional coefficient. According to the actual demand, the proportional $\operatorname{link} K_{a}$ can be taken as a simplified link of the servo amplifier, and $K_{a}=0.006 \mathrm{~A} / \mathrm{V}$. Then as a second-order oscillation link, the transfer function of the servo valve is $\mathrm{kp}$

$$
G_{s v}(s)=\frac{K_{s v}}{\frac{s^{2}}{\omega_{s v}^{2}}+\frac{2 \xi_{s v}}{\omega_{s v}} s+1}
$$

In the above formula, $\xi_{s v}$ is the damping ratio of servo valve. According to the field situation, let $\xi_{s v}=0.6 ; K_{s v}$ is the flow gain of servo valve, and its calculation result is $K_{s v}=0.0665 \mathrm{~m}^{3} / s \cdot A ; \omega_{s v}$ is the natural frequency of servo valve, and its value is $\omega_{s v}=754 \mathrm{rad} / \mathrm{s}$. The controlled object and hydraulic cylinder can be regarded as the combination of second-order link and integral, and its transfer function is:

$$
G_{h}(s)=\frac{1 / A_{p}}{s\left(\frac{s^{2}}{\omega_{h}^{2}}+\frac{2 \xi_{h}}{\omega_{h}}+1\right)}
$$

In the above formula, $\xi_{h}$ is the hydraulic damping ratio, and $\xi_{h}=0.2$ and $\omega_{h}$ are taken as the hydraulic natural frequency of the valvecontrolled cylinder system according to the actual situation, which 
is calculated to be $247 \mathrm{rad} / \mathrm{s}$, and the effective area $A_{p}$ of the hydraulic cylinder is $0.0218 \mathrm{~m}^{2}$.

\subsection{Algorithm control process}

The hybrid algorithm mainly involves the following parameters: individual fitness, initial temperature, annealing rate, acceptance probability of new individuals during annealing, genetic operator value, and determination of individual fitness. Generally, the objective function of the problem is used as a formula for calculating individual fitness.

The annealing rate is the decisive factor to ensure the convergence of the algorithm. If the temperature drops too fast, the extreme value may be lost, but if the temperature drops too slowly, the convergence of the algorithm will be greatly reduced. Therefore, in order to ensure the superiority of the simulated annealing algorithm, the annealing rate must be determined by combining the initial temperature with the exponential function, that is, the initial temperature is used as the main parameter in the first half, while the exponential function is used as an important basis in the second half of optimization.

Acceptance probability means that after genetic operation, for a newly generated individual, a value will be generated due to the difference and uncertainty brought about by the subsequent operation, so as to determine whether the newly generated individual is suitable for the next operation.

There are three basic operations in GA: selection, crossover and mutation. there are two important parameters in the evolution process: the selection of crossover probability $P_{c}$ and mutation probability $P_{m}$ is the key to affect the behavior and performance of GA. therefore, based on the basic GA, an algorithm is designed to make $P_{c}$ and $P_{m}$ change adaptively.

According to the above analysis, the crossover probability is mainly changed adaptively:

$$
P_{c}= \begin{cases}P_{c 1}-\frac{\left(P_{c 1}-P_{c 2}\right)\left(f^{\prime}-f_{\min }\right)}{f_{a v g}-f_{\min }} & f^{\prime}<f_{a v g} \\ P_{c 2}-\frac{\left(P_{c 2}-P_{c 3}\right)\left(f^{\prime}-f_{a v g}\right)}{f_{\max }-f_{a v g}} & f^{\prime} \geq f_{a v g}\end{cases}
$$

Where $f_{\max }$ is the maximum fitness value; $f_{a v g}$ is the average fitness value; $f_{\min }$ is the minimum fitness value; $f^{\prime}$ is a larger fitness value among crossover individuals. $f$ is the fitness value of the variant individual; $P_{c 1}, P_{c 2}, P_{c 3}$ is a constant and is adjusted in optimization.

The control process of hybrid algorithm based on path optimization is as follows:

(1)According to the description of the actual problem and the constraint conditions of the problem, a population of $n$ individuals is randomly generated;

(2)Calculating the fitness value of each individual according to the objective function;

(3)Calculating annealing temperature;

(4)Calculating the fitness value of newly generated individuals in the population;

(5)Calculating the acceptance probability of an individual and annealing;

(6) Generating the next generation of individuals according to the crossover and mutation rules in GA;

(7)Update the whole population;

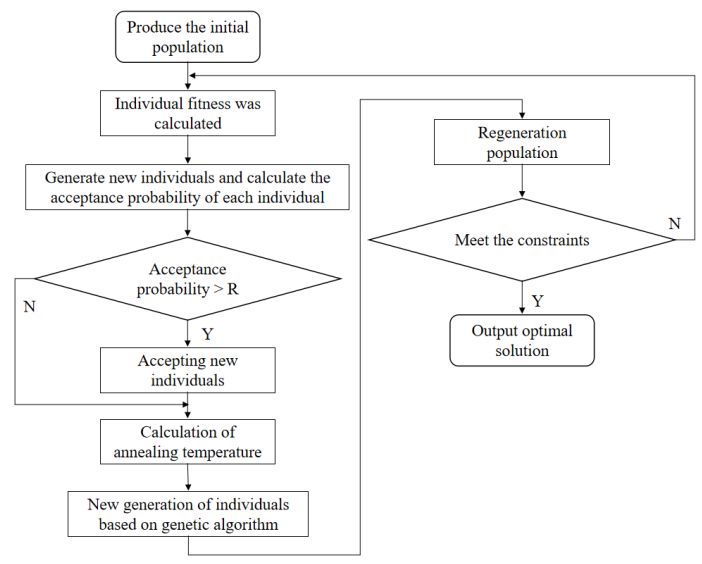

Figure 4: Algorithm flow chart

Table 1: Comparison of test results

\begin{tabular}{lll}
\hline Algorithm & Average time taken (s) & Optimal result \\
\hline GA & 1.53 & 605 \\
Basic ACA & 0.88 & 488 \\
Path optimization & 0.81 & 451 \\
hybrid algorithm & & \\
\hline
\end{tabular}

(8)Judge whether the end condition is met. If yes, output the running result; otherwise, go to (1) and (2) to continue the operation until the ideal solution is reached.

The algorithm uses the number of iterations as the end condition, and outputs the results in the 50th generation. The algorithm flow is shown in Figure 4 below:

\section{EXPERIMENTAL ANALYSIS}

Through the above algorithm design, use Microsoft Visual $C++6.0$ to edit. The algorithm has friendly software interface, simple operation method, stable software operation and high reliability. It can be used practically and as an experimental verification tool. This software integrates three algorithms: GA, ACA and hybrid algorithm of ant colony and genetic fusion. The lower half of the interface is the coordinate of convergence curve. The software can use any one of the algorithms to process data according to requirements, and can also compare the advantages and disadvantages of different algorithms for the same data. When analyzing the path, the nodes that the path passes through are converted into plane coordinates, and the data is loaded by import and the points on the plane are converted on the interface. In this software, there is a parameter setting interface, in which the values of related parameters can be set. The algorithm test results are shown in Table 1

Compared with GA and basic ACA, the improved hybrid algorithm obviously shortens the search time, converges faster than genetic algorithm and ACA, and finds the shortest path. Through the analysis and comparison of experimental results, this algorithm has certain advantages in time efficiency and quality of solving problems. 


\section{CONCLUSION}

Under the influence of global economy, electrical automation plays an increasingly important role in our national economy. The electrical automation control system has completely changed the traditional substation management mode. The advanced and scientific electrical automation control system can ensure the safe and reliable distribution and minimize the occurrence of power failures. In this paper, an electrical automation control system based on path optimization hybrid algorithm is designed. The optimal design method of power control system can shorten the calculation amount, has strong operability and simplifies the parameter setting process of PID controller. Experiments show that the hybrid algorithm is effective in solving electrical automation control problems, and the algorithm is simple and easy to operate. By improving the initial population of GA, the solution efficiency and quality of GA are improved.

\section{REFERENCES}

[1] Wanghaiyue. Application and Development Trend of Electrical Automation Control System. World nonferrous metals, vol. 000, no. 004, pp. 287,289, 2019.
[2] Zhou Jingyu. Application of PLC Technology in Electrical Engineering and Its Automation Control System. Electric power system equipment, vol. 000, no. 010, pp. 66-67, 2019.

[3] Caldeira T, Ciarelli P M, Neto G A. Industrial Optical Character Recognition System in Printing Quality Control of Hot-Rolled Coils Identification. Journal of Control, Automation and Electrical Systems, vol. 31, no. 1, pp. 108-118, 2020.

[4] Hacene N, Mendil B. Fuzzy Behavior-based Control of Three Wheeled Omnidirectional Mobile Robot. International Journal of Automation and Computing, vol. 16, no. 02 , pp. 37-59, 2019.

[5] Zheng D, Zhang H, Andrew Zhang, et al. Consensus of the Second-order Multiagent Systems under Asynchronous Switching with a Controller Fault. International Journal of Control, Automation and Systems, vol. 17, no. 1, pp. 136-144, 2019.

[6] X Gao, W Zhao, S Wang,et al. A Prescribed Performance Adaptive Control for Hysteresis Hammerstein System. Journal of Systems Science and Complexity: English Edition, vol. 032, no. 004, pp. 1039-1052, 2019.

[7] ChaoHuang, FazelNaghdy, HaipingDu,et al. Shared Control of Highly Automated Vehicles Using Steer-By-Wire Systems. Acta automatica sinica: English version, no. 2, pp. 410-423, 2019.

[8] Bai K, Yildizbasi A. Optimal Siting and Sizing of Battery Energy Storage System for Distribution Loss Reduction Based on Meta-heuristics. Journal of Control, Automation and Electrical Systems, vol. 31, no. 6, pp. 1469-1480, 2020.

[9] Anand I, Agarwal D, Senthilkumar S, et al. A Dynamic Load Controller for a Standalone Solar PV System Employing a Dual Input/Output Biphase dc-dc Converter. Journal of Control, Automation and Electrical Systems, vol. 30, no. 5, pp. 812-821, 2019. 